LB Lawrence Berkeley Laboratory UNIVERSITY OF CALIFORNIA
Accelerator \& Fusion , $\quad$ BLL-- 19602
Research Division
DE85 016553

presented at the 1985 Particle Accelerator Conference, Vancouver, B;.C. Canada, May 13-16; 1985

THE HIGH TEMPERATURE EXPERIMENT.FOR ACCELERATOR INERTIAI FUSION

E.P. Lee

MaY 1985

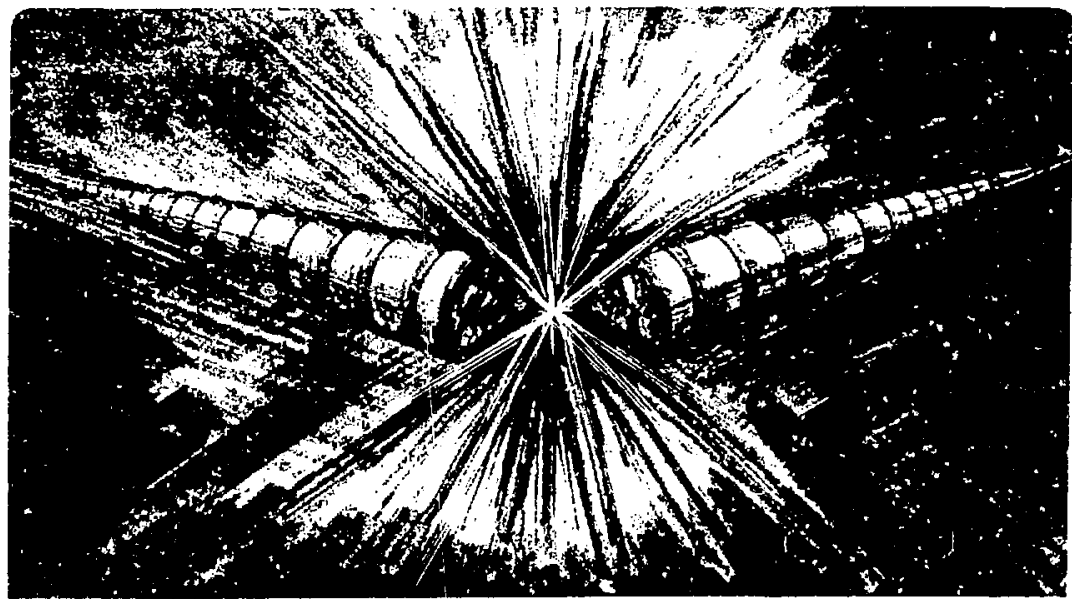

Prepared for the U.S. Department of Energy under Contract DE-AC03-76SF00098 


\title{
DISCLAIMER
}

This report was prepared as an account of work sponsored by an agency of the United States Government. Neither the United States Government nor any agency thereof, nor any of their employees, makes any warranty, express or implied, or assumes any legal liability or responsibility for the accuracy, completeness, or usefulnass of any information, apparatus, produnt, or process disclosed, or represents that its we would not infringe privately owned rights. Re.er. ence herein to any specific commercial p:oduct, process, or service by trade name, trademaik, manufacturer, or otherwise does not necessarily constitute or imply its endorsement, recosimendation, or favoring by the United States Government or any agency thereof. The vie ws and opinions of authors expressed herein do nol necessarily state or reflect those of the United States Government or any agency thereof.

\section{THE HIGH TEMPERATURE EXPERIMENT FOR ACCELERATOR INERTIAL FUSION*}

\author{
Edward P. Lee
}

\author{
Lawrence Berkeley Laboratory \\ University of California \\ Berkeley, CA 94720
}

May 1985

* This was suppor':ed by the Office of Energy Research, Office of Basic Energy Sciences, U.S. Department of Energy under Contract DE-AC03-76SF00D98. 


\author{
Edward P. Lee \\ Lawrence Berkeley Laboratory \\ University of California \\ Berkeley, California 9472D
}

\section{Abstract}

The High Temperature Experiment (HTE) is intended to produce temperatures of $50-100 \mathrm{eV}$ in solid density targets driven by reavy ion beams from a multiple beam induction linac [1]. The fundamental variables (particle species, energy, number of beamlets, current and pulse length) must be fixed to achieve the temperature at minimum cost, subject to criteria of technical feasibility and relevance to the development of a Fusion Driver. The conceptual design begins with an assumed (radiationlimited) target temperature and uses limitations due to particle range, beamlet perveance, and target disassembly to bound the allowable values of mass number $(A)$ and energy (E). An accelerator model is then applied to determine the minimum length accelerator, which is a guide to total cost. The accelerator model takes into account limits on transportable charge, maximum gradient, core mass per linear meter, and head-to-tail momentum variation within a pulse.

For specified target temperature, T, spot size $r$ and beamlet number $N$, the particle energy $E$ must lie above a critical value which only weakly depends on $A$ $\left(\mathrm{E}_{\mathrm{Cr} t}{ }^{\circ} \mathrm{A} \cdot \mathrm{C}\right)$. The minimum length accelerator is associated with the high mass end of this line, determined by a condition on range in the target. Singly charged potassium ions with $E=132 \mathrm{MeV}$ are a good match to an 80 ev temperature.

\section{Tarqet Madel}

The heated talget is taken to be a cylinder of felted Aluminum ( $\rho \leqslant .1 \mathrm{gm} / \mathrm{cm}$ ) with diameter and length equal to the diameter of a focussed beamlet (see Fig. 1). The heating equation for the target is

\section{HTE Target}

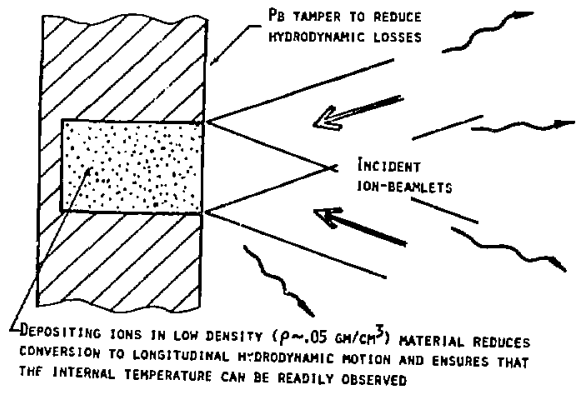

$$
\frac{d e}{d t}=\frac{S-\sigma T^{4}}{R} \text {. }
$$

where e $\propto T^{1.5}$ is specific energy, $S$ is beam irradiance, and $R$ is particle range. For final temperature $T_{f} \approx 50-100 \mathrm{eV}$, forward emitted radiation is the dominant mechanism for energy loss, so the irradiance is set high enough to achieve a balance:

$$
S=\sigma_{f}^{4}=\left(\frac{T_{f}}{55.8 \mathrm{eV}}\right)^{4} \frac{T \mathrm{H}}{\mathrm{cm}^{2}} .
$$

To reach this temperature the specific energy e( $\left.T_{f}\right)$ must be supplied. This condition defines a scale time for heating $\left(\tau_{H}\right)$, which we set equal to the beam pulse length

$$
\tau_{p}=\tau_{H}=\frac{3}{2} \frac{c\left(T_{f}\right) R}{\sigma T_{f}^{4}} .
$$

When the time $\left(\tau_{H}\right)$ is reached the temperature equals $96 \%$ of $T_{f}$, and the beam pulse ends. For further details of this model see ref. [2].

it is clear that a reduction in range (measured in $\mathrm{gm} / \mathrm{cm}^{2}$ ) allows a reduction in $\tau p$, which could lead to a savings in accelerator cost. A 'lower limit on range, $R=.004 \mathrm{gm} / \mathrm{cm}^{2}$, is imposed here because stripping of beam ions in the target would otherwise be very incomplete and the energy deposition would not resemble that of fusion driver; the significance of HTE would be diminished. The ions considered for HTE have mass numbers $A \approx 10$ to 50 and energy $E \approx 40$ to $200 \mathrm{MeV}$. A good formula for the rarige of these ions in heated Aluminum is

$$
R=.004\left(E_{H e V}^{3 / 2}\right) / A^{2}\left\langle g m / \mathrm{cm}^{2}\right\rangle
$$

A second target-related feature which limits the choice of beam parameters is target disassembly. If heating is too slow then there is time for the felted Al to extrude forwards from its enclosure as an expansion wave (see $\mathrm{Fig.} \mathrm{2).} \mathrm{A} \mathrm{criterion} \mathrm{for} \mathrm{avoiding} \mathrm{target} \mathrm{degradation} \mathrm{by} \mathrm{this}$ mechanism is that the pulse length be shorter than twice the time required for a sound wave to reach the backside of the (half) heated target cylinder. Hence we define a disassembly time

$$
\tau_{D}=\frac{2 r}{c_{s}} \approx \frac{(5790 \mathrm{~ns}) 2 r_{c m}}{\sqrt{T_{f}\left(2^{\star}+1\right)}},
$$

where $Z^{*}$ is the final target ionization state (at $T_{f}$ ), and the criterion is

$$
T_{D}<2 T_{D}
$$

Fig. 1 . 


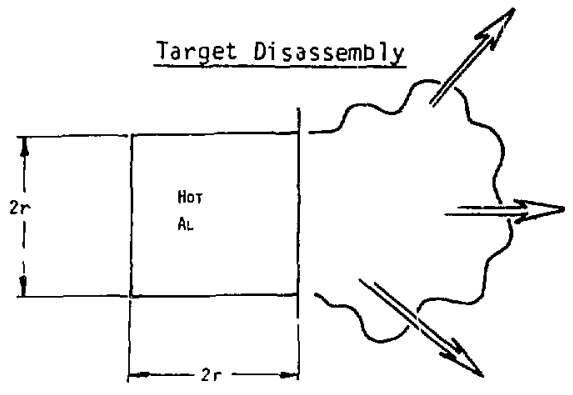

, $4565,2,35 ?$

Fig. 2.

\section{Perveance Condition}

A third constraint which restricts beam parameters is the allowable perveance (per beamlet) in the final focus and transport zone. This is a limit on the line charge or current which can be precisely aimed and focussed. We define perveance per beamlet as

$$
K_{0}=\frac{2 I_{0}}{0^{3} A\left(31 \times 10^{6} \text { AmD }\right)}
$$

If $k$ is sufficiently large $\left(\gg 10^{-4}\right)$ then a high Gegree of neutralization must be present during final transport to achieve the desired small spot radius on the target. In this situation the focal system is designed with compensation for high current effrcts, but it then is sensitive to moderate variations of current within a pulse. A rough criterion is adopted here:

$$
k_{0} \leq 2 \times 10^{-4}
$$

which corresponds to a required charge neutralization fraction $F_{e} \geq .7$ in final transport, assuming the beamlet cone angle is at the aberration limit $\theta=20 \mathrm{mr}$.

The perveance condition is readily converted to a condition on $T, E, A$ and $N$ by substituting from Eq. (2) for $S=N E I_{0} / \pi r^{2}$ and $B=\left(2 E / m c^{2}\right)^{2 / 2}$.

\section{Boundaries in the $(A, E)$ Plane}

The limits placed on range, disassembly time and perveance may be written respectively

$$
\begin{gathered}
\frac{E^{3 / 2}}{A^{2}}>1.0 . \\
\frac{E^{3 / 2}}{A^{2}}<\left(1.464 \times 10^{-3}\right)\left(\frac{r}{.1}\right)\left(\frac{T_{f}^{2}}{\sqrt{2^{\star}+1}}\right)^{1.74} . \\
\frac{E^{5 / 2}}{A^{1 / 2}}>\left(6.552 \times 10^{-4}\right) T_{F}^{4}\left(\frac{r}{.1}\right)^{2}\left(\frac{16}{H}\right) .
\end{gathered}
$$

where the units of $E, T_{f}$, and $r$ are $\mathrm{MeV}, \mathrm{eV}$ and $\mathrm{cm}$. The allowed zone of the $E, A$ plane is shown in $F$ ig. (i) for $T_{f}=B D \mathrm{eV}$, along with the computed pulse length on the high and low A boundaries. An extremely significant point is that $A$ can be increased through a large range of values along the perveance dominated boundary with only a small increase in $E$ [note $(d \log E) /(d \log A)=1 / 5]$. A consequence is that a short pulse length and low total pulse energy $W$ can be realized with high $A$, with only a small penalty in increased $E$. The low cost accelerator systems are expected to correspond to short pulse lengths, provided the match to pulsed power technology is feasible.

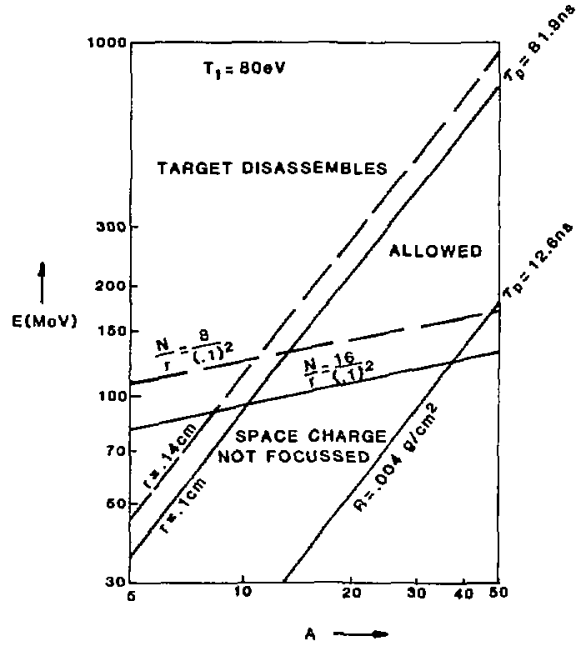

XEL $855-239$ ?

Fig. 3. Energy and mass number boundaries for an Al target heated to $\mathrm{BD} \mathrm{eV}$. The high $\mathrm{A}$ are excluded by shortness of range, low $A$ are excluded by target disassembly, and low $E$ are excluded by the perveance condition.

\section{Accelerator Model}

Tarjet heating considerations and the perveance limit provide a range of mass numbers and energies available to reuch a specified final temperature. From these parameters, and a spot size $(r \approx .1 \mathrm{~cm}$ estimated from chromatic effects), we can calculate the total charge delivered by the accelerator system. These final beam parameters, along with several stipulated accelerator feitures can be used to determine the minimum accelerator length, which is a guide to total ost.

Total length $\left(Z_{A}\right)$ is minimized by initial injection at a high kinetic energy; we adopt the value $E_{0}=2 \mathrm{MeV}$ in all examples. At this energy, the maximum transportable line charge density is about $.095 \mu \mathrm{C} / \mathrm{m}$ in an electrostatic alternating gradient lattice filled to half aperture [3]. For an assumed number of beamlets ( $N$ \} we can then calculate the length of the initial pulse $?_{0}$ (in meters).

Acceleration is carried out at the highest gradient consistent with the following considerations, which dominate in the given order as energy increases: 
a. The velocity tilt at any given position ( $z$ ) does not exceed $A B / O=.2$. Higher values result in an expanded pulse tail which exceeds the aperture criterion.

b. The product of gradient and pulse length does not exceed $\omega_{m}=.45(\mathrm{~V}-\mathrm{s}) / \mathrm{m}$. This is imposed to keep induction core mass per meter within reasonable bounds.

c. The maximum gradient is $\varepsilon_{\mathrm{m}}=.43 \mathrm{MV} / \mathrm{m}$, characteristic of long pulse LIA systems.

For short pulse lenoths, corresponding to the upper limits on mass number, the accelerator length is dominated by a combination of flux $\left(\sigma_{0}\right)$ and gradient $\left(\varepsilon_{n}\right)$ limited zones. However for low values of $A$, the pulse is very long $\left(\varepsilon_{0} \sim 100 \mathrm{~m}\right)$ and the flux limited zone becomes large. Table 1 gives a summary of these calculations.

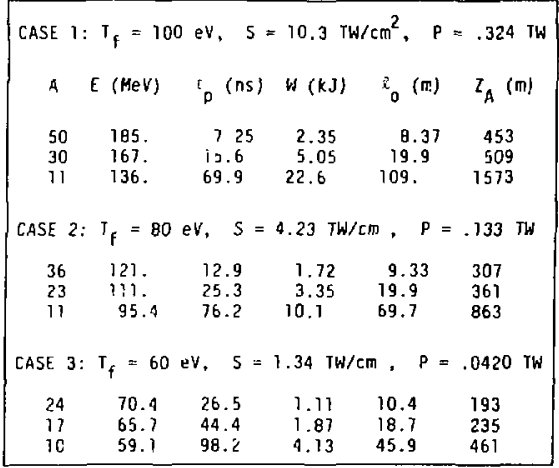

Table 1. Major system parameters are tabulated. For each value of $T_{f}$, three points in the $(A, E)$ plane are examined. These points lie on the maximum perveance line, with the largest value of A close to the minimum range boundary and the smallest value of $A$ close to the disassembly boundary. In all cases we assume $H=16$ and $r=.1 \mathrm{~cm}$.

\section{Canclusions}

This HIE study has identified the following significant points, which guide the selection of major parameters:

1. Multiple beamlets are needed to keep the final perveance and initial pulse length withirt reasonable bounds.

2. High mass ions match short pulse lengths and ranges, with a small penalty in increased energy. A lower bound on pulse length $\left(\tau_{p} \propto R T^{-5 / 2}\right.$ ) is found in this limit.

3. Low mass ions match long pulse lengths with a small decrease in kinetic energy. Targat disassembly is important for low $T_{f}$.
4. Emittance does not determine spot size if a high brightness source is available, and there is no emittance dilution during acceleration.

5. A simple accelerator model gives reasonable agreement with more detalled HTE reference designs [4].

\section{References}

[1] T.J. Fessenden, "Induction Linacs for Heavy Ion Fusion Research," Proceedings of the 1984 Linear Accelerator Conference, Seeheim/Darmstadt, West Germany, May 7-11, 1984.

[2] E.P. Lee and J. W-KMark, "Spot Heating Calculations for a Heavy Ion Driven High Temperature Experiment," LBL-16890, April 1984.

[3] E.P. Lee, T.J. Fessenden, and L.J. Laslett, "Transportable Charge in a Periadic Alternating Gradient System," included in these proceedings.

[4] C.H. Kim and L. Smith, "A Design Procedure for Acceleration and Bunching in an Ion Induction Linac, LBL-19137, February 1985, submitted to Particle Acceleratars. 
This report was done with support from the Department of Energy. Any conclusions or opinions expressed in this report represent solely those of the author(s) and nat necessarily those of The Regents of the University of Califomia, the Lawrence Berkeley Laboratory or the Department of Energy.

Reference to a company or product name does not imply approval or recommendation of the product by the University of California or the U.S. Depars ment of Energy to the exclusion of others that may be suilable. 